\title{
Short communication: Complete genome sequence of Lactobacillus plantarum J26, a probiotic strain with immunomodulatory activity
}

\author{
Ziwei Zhang, ${ }^{*}$ () Chaoxin Man, ${ }^{*}$ - Linlin Sun, Xinyan Yang, Mingyu Li, Wei Zhang, and Yujun Jiang† $\odot$ \\ Key Laboratory of Dairy Science, Ministry of Education, Department of Food Science, Northeast Agricultural University, Harbin, 150030, China
}

\begin{abstract}
Lactobacillus plantarum J26, a significant probiotic isolated from Chinese traditional fermented dairy products, exerts a positive immunomodulatory effect by regulating the expression of immune-related genes. We

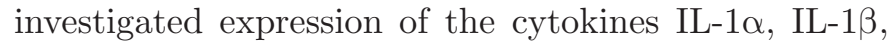
IL-6, and tumor necrosis factor- $\alpha$ in the intestinal tract of mice stimulated by L. plantarum J26. In vivo, these cytokines were upregulated, peaked on d 5 , and then decreased to the control level, indicating that L. plantarum J26 could induce expression of the genes encoding these proinflammatory cytokines. Teichoic acids produced by L. plantarum are recognized as key immunomodulatory molecules involved in the regulation of the host immune response. To better understand the genetic basis of this immunomodulatory mechanism, we sequenced and analyzed the whole genome of $L$. plantarum J26. The genome of L plantarum J26 contains a circular chromosome and 4 circular plasmids. Lactobacillus plantarum J26 was predicted to synthesize ribitol-type backbones of wall teichoic acid. Furthermore, orthologous average nucleotide identity (OrthoANI) values showed that the genome was highly similar $(>98.00 \%)$ to other L. plantarum strains, especially to L. plantarum ST-III and JDM1. The genomic data of L. plantarum J26 provide a genetic basis to further elucidate its mechanism of immunoregulation and will facilitate its application in the functional dairy food industry.
\end{abstract}

Key words: Lactobacillus plantarum J26, complete genome sequence, immunomodulatory activity, cytokine

\section{Short Communication}

Lactobacillus constitutes a large and diverse genus within the lactic acid bacteria and its genetic diversity is larger than that of a normal family (Sun et

Received March 10, 2019

Accepted July 30, 2019.

${ }^{*}$ These authors contributed equally to this work.

†Corresponding author: yujun_jiang@163.com al., 2015). Lactobacillus plantarum is a gram-positive bacterium (Hugenholtz, 1998) that inhabits relatively abundant ecological niches and plays a significant role in food microbiology and human nutrition because of its fermentative and probiotic functions (Salvetti et al., 2012). Recently, numerous studies have investigated the health benefits of $L$. plantarum, such as effects on oxidative stress regulation ( $\mathrm{Li}$ et al., 2014), reduction of cholesterol level (Barreto et al., 2014), and management of bacterial composition in feces (Goossens et al., 2003). Among these properties, the potential of $L$. plantarum to inhibit inflammation has received extensive attention. Lactobacillus plantarum GB-LP2 has also shown antiviral effects against the influenza virus in mice (Choi et al., 2015). Similarly, L. plantarum PS128 reduced LPS-induced proinflammatory cytokine production in a mouse macrophage cell model (Liu et al., 2015).

Teichoic acid (TA), the major component of cell walls of gram-positive bacteria, is recognized as key immunomodulatory molecule involved in the regulation of host immune response. For example, heat-killed $L$. plantarum MYL26 and bacterial cell wall extracts are able to reduce LPS-induced inflammation by impairing toll-like receptor 4 (TLR4)-nuclear factor $\kappa \mathrm{B}(\mathrm{NF}-\kappa \mathrm{B})$ signal transduction in vitro, suggesting that constituents of bacterial cell wall help attenuate inflammation (Chiu et al., 2013). In addition, an investigation found that D-alanylation of TA abolished the pro- and anti-inflammatory response in vivo (Smelt et al., 2013). However, immunomodulatory capability varies in different strains, probably because the types and structures of TA are significantly different (Han et al., 2003).

Lactobacillus plantarum J26 is a common probiotic strain isolated from traditional fermented dairy products. Our recent investigation showed that strain J26 could alleviate oxidative stress by modulating the production of antioxidant enzymes significantly in vitro (Hou et al., 2019). In the current study, we confirmed that the strain exhibits potent immunomodulatory activity. To better understand the immunomodulatory mechanism of L. plantarum J26 and obtain detailed 
insight into the genetic basis, we analyzed the whole genome sequence of $L$. plantarum J26 combined with biochemical assays.

Lactobacillus plantarum J26 was isolated from Chinese traditional fermented dairy products in Inner Mongolia (China). It was grown in de Man, Rogosa, and Sharpe (Qingdao Hope Bio-Technology Co. Ltd., Qingdao, China) broth at $37^{\circ} \mathrm{C}$ for $16 \mathrm{~h}$ through 2 propagation steps. Genomic DNA was extracted using the Qiagen DNA Mini kit (Qiagen, Hilden, Germany) following the manufacturer's instructions.

Quantity and quality determinations of genomic DNA in L. plantarum J26 were conducted by Qubit 2.0 (Thermo Fisher Scientific, Waltham, MA) and Agilent 2100 Bioanalyzer (Agilent Technologies, Santa Clara, $\mathrm{CA}$ ). The genomic DNA was sequenced by Illumina Miseq (insert size of 400 bp; Illumina Inc., San Diego, $\mathrm{CA}$ ) with paired-end sequencing mode and Pacbio sequencing (20,000 bp template library; Pacific Biosciences, Menlo Park, CA) with standard sequencing mode using next-generation sequencing technology and single molecule real-time (SMRT) technology. A total of 3,221,902 reads were obtained. Genome assembly was performed using A5-miseq v20150522 (Coil et al., 2015) and CANU (Koren et al., 2017) software for the data obtained by second- and third-generation sequencing platforms. Then, the assembled results were confirmed by MUMmer software (http://mummer.sourceforge .net/) to determine the positional relationship between contigs and to fill gaps (Delcher et al., 2003). Finally, a complete genome without gaps was constructed after the correction using Pilon software (Walker et al., 2014). Gene prediction was carried out by GeneMarkS (http://topaz.gatech.edu/). The predictions of transfer (t)RNA, rRNA, and small (s)RNA were identified using tRNAscan-SE (Lowe and Eddy, 1997), rRNAmmer (Lagesen et al., 2007), and Rfam (Gardner et al., 2009) softwares, respectively. Genome annotation was accomplished by National Center for Biotechnology Informa- tion (NCBI) Prokaryotic Genome Annotation Pipeline (Pruitt et al., 2012) and confirmed by BLAST analysis (www.ncbi.nlm.nih.gov/protein). Using the multiple sequence alignment software MUSCLE (https://www .ebi.ac.uk/Tools/msa/muscle/) to align the core gene sequences of the L. plantarum J26 and other species, we constructed a phylogenetic tree based on the core genes (Kumar et al., 2016). Furthermore, the average nucleotide identity of the genome sequence between the L. plantarum J26 and other reference strains was evaluated by orthologous average nucleotide identity (OrthoANI; http://www.ezbiocloud.net/tools/orthoani) tool.

The expression of immune-related genes was evaluated in vivo. All of the procedures used here were approved by the Institutional Animal Care and Use Committee of Northeast Agricultural University in China, and the animal experiments were performed following the Northeast Agricultural University guidelines for Laboratory Animals Care and Use.

Forty BALB/cl mice, 8 wk old and 18 to $22 \mathrm{~g}$ of BW, were provided by Heilongjiang Province Medical University Laboratory Animal Center (Harbin, China). Mice were randomly divided into 2 groups and acclimated in pathogen-free cages under controlled conditions $\left(22^{\circ} \mathrm{C} \pm 2^{\circ} \mathrm{C}\right.$, relative humidity $55 \% \pm 5 \%, 12$-h light/ dark cycles) with free access to water and a standard diet for $7 \mathrm{~d}$. After this period, the experimental group was given $0.3 \mathrm{~mL}$ of skim milk containing L. plantarum J26 $\left(10^{8} \mathrm{cfu} / \mathrm{mL}\right)$ each day. The mice in the control group were given skim milk without L. plantarum J26. The mice were killed at $1,3,5$, or $7 \mathrm{~d}$ after supplementation of bacteria. The colon and ileum tissue were removed, washed with cold saline, and placed in liquid nitrogen (Jiang et al., 2016). To evaluate the mRNA expression levels of IL1A,IL1B,IL6, and TNFA, RNA of colon and ileum was extracted using a commercial kit (RNAprep Tissue Kit, Tiangen Biotech, Shanghai, China) and converted to cDNA using a PrimeScript RT

Table 1. The base sequence, annealing temperatures, and sizes of PCR products for target gene-specific primers

\begin{tabular}{|c|c|c|c|}
\hline $\begin{array}{l}\text { Target } \\
\text { gene }\end{array}$ & Primer sequence $^{1}\left(5^{\prime} \rightarrow 3^{\prime}\right)$ & $\begin{array}{c}\text { Annealing } \\
\text { temperature }\left({ }^{\circ} \mathrm{C}\right)\end{array}$ & $\begin{array}{l}\text { Product } \\
\text { size (bp) }\end{array}$ \\
\hline \multirow[t]{2}{*}{$G A P D H$} & F: GCCTGGAGAAACCTGCC & 55.4 & \multirow[t]{2}{*}{200} \\
\hline & R: ATACCAGGAAATGAGCTTGACA & 56.7 & \\
\hline \multirow[t]{2}{*}{$I L 1 A$} & F: TCTGCCATTGACCATCTC & 50.7 & \multirow{2}{*}{183} \\
\hline & R: AATCTTCCCGTTGCTTG & 50.8 & \\
\hline \multirow[t]{2}{*}{$I L 1 B$} & F: AAGTTGACGGACCCCA & 50.6 & \multirow[t]{2}{*}{126} \\
\hline & R: GTGATACTGCCTGCCTGA & 51.3 & \\
\hline \multirow[t]{2}{*}{ IL6 } & F: GAAGTGATTCTTACGCA & 42.5 & \multirow[t]{2}{*}{247} \\
\hline & R: GTTTAGGTGGAGGTGTC & 43.0 & \\
\hline \multirow[t]{2}{*}{ TNFA } & F: CGTAGCAAACCACCAAG & 49.3 & \multirow[t]{2}{*}{149} \\
\hline & R: CCGTGAAGAGAACCTGG & 50.4 & \\
\hline
\end{tabular}

${ }^{1} \mathrm{~F}=$ forward; $\mathrm{R}=$ reverse. 

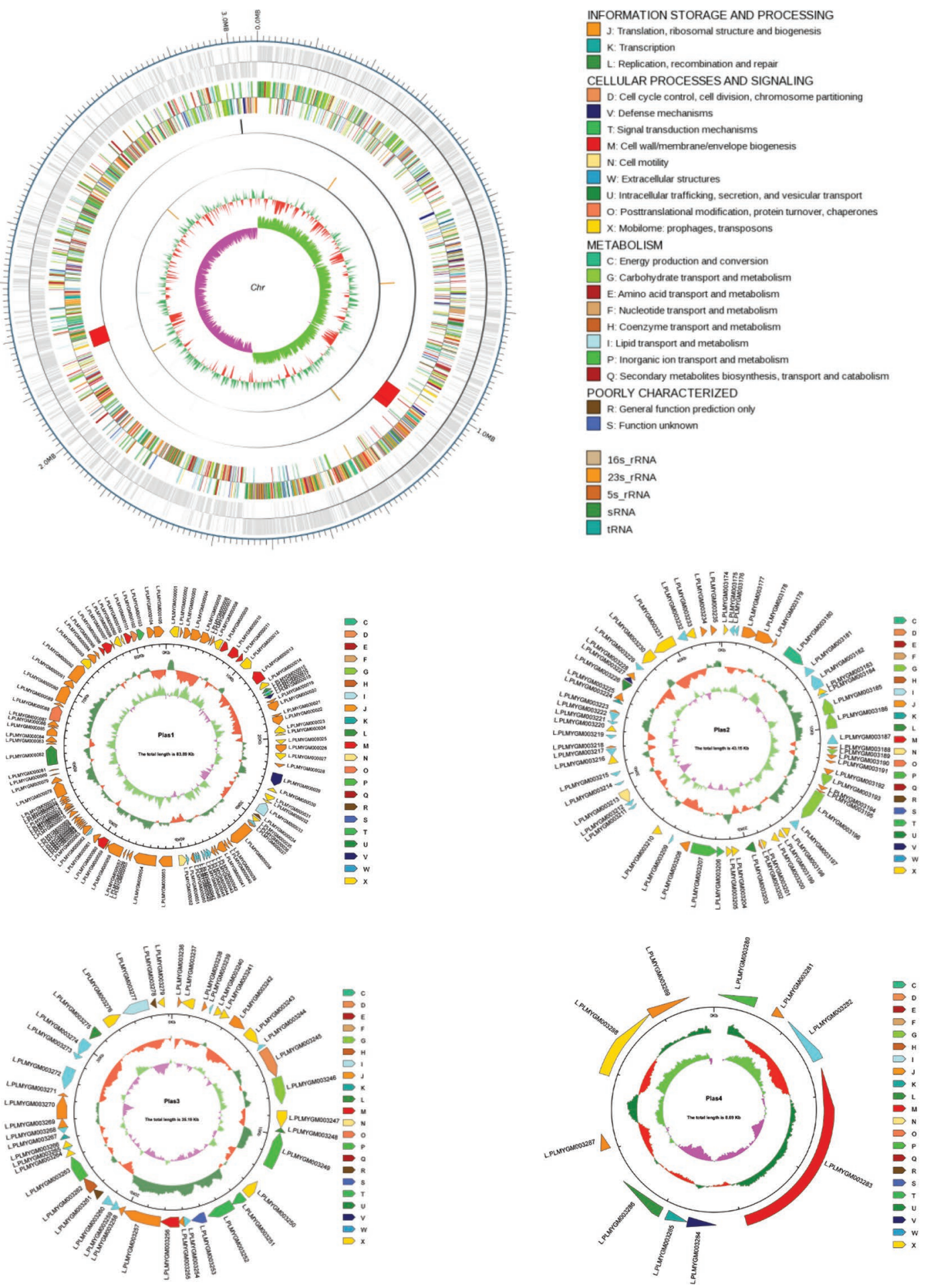

Figure 1. Circular genome map of Lactobacillus plantarum J26. From periphery to center: genome sequence (ring 1 and 2), Cluster of Orthologous Groups of proteins (COG) annotated coding sequences (ring 3 and 4), intact prophages in red and clustered regularly interspaced short palindromic repeats (CRISPR) in black (ring 5), noncoding (nc)RNA genes (ring 6), GC content (ring 7), and GC skew [(G+C)/(G+C)] (ring 8 ), where values $>0$ are in green, and values $<0$ are in purple. The smaller maps show the genome map of plasmids in $L$. plantarum J26. The image was created using the software Circos (http://circos.ca/software/download/circos/). 
Table 2. Features of Lactobacillus plantarum J26 genome

\begin{tabular}{lcccc}
\hline Feature $^{1}$ & Chromosome & Plasmid 1 & Plasmid 2 & Plasmid 3 \\
\hline Size (bp) & $3,096,468$ & 83,889 & 43,153 & 35,191 \\
GC content (\%) & 44.79 & 41.14 & 40.30 & 45.48 \\
Predicted genes & 3,068 & 105 & 62 & 44 \\
Protein-coding genes (CDS) & 3,068 & 105 & 62 & 35.986 \\
rRNA operons & 16 & 0 & 0 & 10 \\
tRNA & 71 & 0 & 0 & 0 \\
sRNA & 1 & 2 & 1 & 0 \\
\hline
\end{tabular}

${ }^{1} \mathrm{CDS}=$ coding sequence; $\mathrm{tRNA}=$ transfer $\mathrm{RNA} ; \mathrm{sRNA}=$ small RNA sequences.

reagent kit (Takara, Dalian, China). The expression of immune-related genes was analyzed by using real-time quantitative PCR (ABI Prism 7500 system; Applied Biosystems, Foster City, CA). Approximately $2 \mu \mathrm{L}$ of the RNA sample was added to the $20-\mu \mathrm{L}$ total reaction volume according to the requirements of the SYBR PremicExTaqTMII (PerfectReal-time) kit (Takara). The GAPDH gene was used as the reference gene. To amplify the selected immune-related genes $I L 1 A, I L 1 B$, IL6, and TNFA and GAPDH, specific primers were designed using Primer 5.0 software (Premier Biosoft, USA; http://www.premierbiosoft.com/primerdesign/ index.html) as shown in Table 1. The protocol for the amplification reactions was as follows: $95^{\circ} \mathrm{C}$ for $30 \mathrm{~s}$ (denaturation) and 40 cycles of $95^{\circ} \mathrm{C}$ for $5 \mathrm{~s}$ and $60^{\circ} \mathrm{C}$ for $34 \mathrm{~s}$ (amplification and quantification). The results were expressed as relative values after normalization to GAPDH mRNA (Jiang et al., 2016).

The complete genome of $L$. plantarum J26 comprises a circular chromosome $(3,096,468 \mathrm{bp})$ and 4 circular plasmids: pJ26p1 (83,889 bp), pJ26p2 (43,153 bp), pJ26p3 (35,191 bp), and pJ26p4 (8,686 bp), with GC

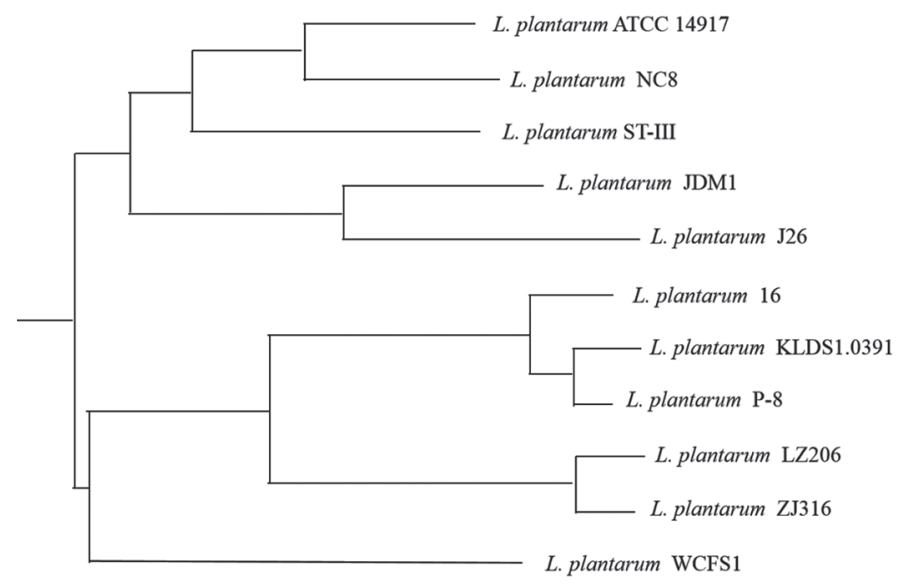

Figure 2. Phylogenetic tree based on core gene sequence showing the phylogenetic relationships of 11 Lactobacillus plantarum strains. Phylogenetic analysis was evaluated by MUSCLE software (https:// www.ebi.ac.uk/Tools/msa/muscle/). contents of $44.79,41.14,40.30,45.48$, and $35.92 \%$, respectively (Figure 1). Among the 3,289 identified protein-coding genes, 3,068 were in the chromosome and $105,62,44$, and 10 in plasmids pJ26p1 to pJ26p4, respectively. Furthermore, the chromosome included 16 rRNA operons, 71 tRNA, and 1 sRNA (Table 2). Each gene was assigned to a functional category according to the Clusters of Orthologous Groups database (www .ncbi.nlm.nih.gov/COG; Table 3). Among 23 functional categories, the most abundant were assigned to transcription (248 genes); 236 genes were assigned to carbohydrate transport and metabolism; and 137 genes were responsible for cell wall, membrane, and envelope biogenesis.

A phylogenetic tree was constructed based on homology of core genes of L. plantarum J26 with related strains. Phylogenetic tree analysis (Figure 2) showed that $L$. plantarum J26 was more closely related to $L$. plantarum JDM1 than to other L. plantarum strains and evolved from the common ancestor compared with ST-III (which contains TA synthesis protein B instead of TA synthesis proteins TagF1 and TagF2 (Liu et al., 2015). The genome of J26 was compared with 10 other L. plantarum strains. The OrthoANI values (Table 4) indicated that the genome of L. plantarum J26 was closest to L. plantarum JDM1 (99.30\% OrthoANI), followed by L. plantarum 5-2 (98.73\%) and L. plantarum WCFS1 $(98.71 \%)$. Generally, two genomes are considered the same species when the ANI value is higher than 95 to $96 \%$ (Lee et al., 2016). Consequently, $L$. plantarum J26 was confirmed to belong to the species of L. plantarum.

Intestinal epithelial cells stimulated by microbes can secrete cytokines and pattern recognition receptors to participate in appropriate immune regulation (Round and Mazmanian, 2009). As shown in Figure 3, we investigated mRNA expression of proinflammatory cytokines IL- $1 \alpha$, IL-1 $\beta$, IL-6, and TNF- $\alpha$ in the intestinal tract of mice stimulated with $L$. plantarum J26. These cytokines were all upregulated, with expression peaking at d 5 , and then decreasing to control levels. Moreover, the expression of $I L 1 A, I L 1 B, I L 6$, and TNFA was sig- 
Table 3. Cluster of Orthologous Groups of proteins (COG) categories of coding proteins in Lactobacillus plantarum J26

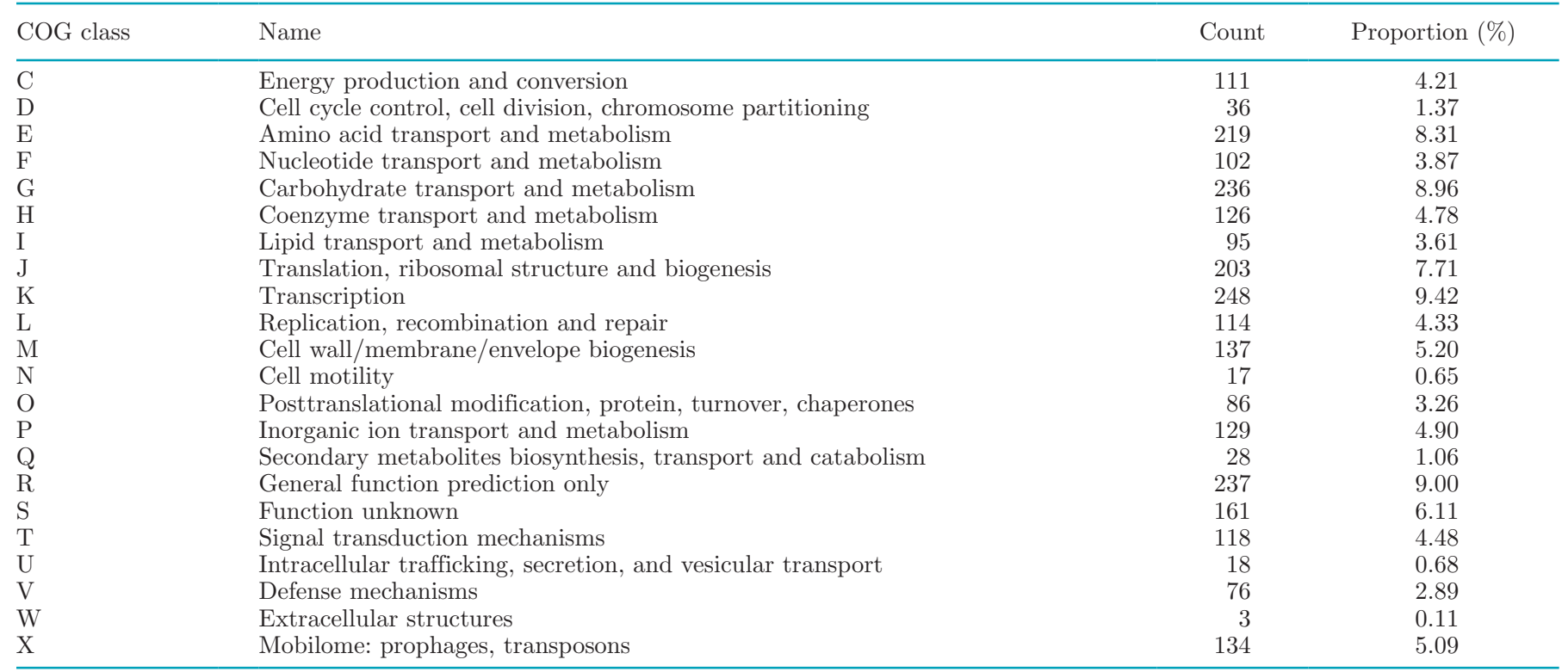

nificantly different from that in the control group $(P<$ 0.01 ) on d 5, indicating that L. plantarum J26 was able to trigger the mRNA expression of the genes encoding these proinflammatory cytokines in vivo, results consistent with our previous study with another L. plantarum

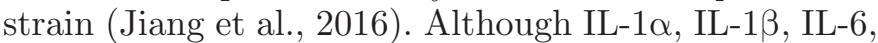
and $\mathrm{TNF}-\alpha$ are considered proinflammatory cytokines, the specific mechanism by which $L$. plantarum J26 regulates their expression is unknown. We found no evidence that the immunomodulatory activity of $L$. plantarum J26 was directly related to certain host cells; our results indicate that the immune responses induced by J26 were likely linked to the type of immune-related cytokines, which is the focus of our further research.

The immunomodulatory mechanism of J26 was further investigated by analysis of the whole genome of $L$. plantarum J26. Teichoic acids consist of 2 types: wall teichoic acid (WTA) and lipoteichoic acid (LTA). For
L. plantarum WCFS1, D-alanylated TA in the cell walls account for several host immunomodulatory effects (Smelt et al., 2013). Because TA are recognized as immunoregulatory molecules, the TA-related genes of $L$. plantarum J26 have been searched by in silico analysis. The synthesis genes encoding the WTA backbones are tag and tar homologs, whereas those of LTA are ltaS. The genes encoding LTA synthase (ltaS), TA glycosylation proteins ( $g t c A 1, g t c A 2, g t c A 3)$, and D-alanylation protein $(d l t X)$ were found in the L. plantarum J26 genome. Two gene regions, tagD1-tagF2 (tagD1, tagF1, and tagF2) and tarI-tarL (tarI, tarJ, tarK and tarL), were regarded as the synthesis genes of glycerol-type and ribitol-type backbones of WTA. In L. plantarum J26, only a glycerol-3-phosphate cytidylyltransferase gene tagD1 was detected. The genes encoding TA synthesis protein $\mathrm{F}$ ( $\operatorname{tagF1}$ and $\operatorname{tagF2}$ ) were absent. However, there are $2 \mathrm{TA}$ synthesis protein $\mathrm{B}$ genes $(\operatorname{tagB1}$

Table 4. Average nucleotide identity (ANI; \%) values between the strains and different Lactobacillus plantarum species

\begin{tabular}{|c|c|c|c|c|c|c|c|c|c|c|c|}
\hline $\begin{array}{l}\text { Strain } \\
\text { code }\end{array}$ & 16 & $5-2$ & JDM1 & LPL-1 & LZ206 & LZ227 & LZ95 & WCFS1 & $\mathrm{J} 26$ & ST-III & ZJ316 \\
\hline 16 & 100.00 & 98.71 & 98.90 & 99.07 & 99.12 & 98.88 & 98.80 & 98.76 & 98.53 & 98.73 & 99.11 \\
\hline $5-2$ & 98.71 & 100.00 & 98.94 & 98.73 & 98.76 & 98.71 & 99.07 & 99.00 & 98.73 & 99.00 & 98.73 \\
\hline JDM1 & 98.90 & 98.94 & 100.00 & 98.87 & 98.72 & 98.83 & 98.96 & 98.95 & 99.30 & 98.87 & 98.75 \\
\hline LPL-1 & 99.07 & 98.73 & 98.87 & 100.00 & 98.70 & 98.80 & 98.74 & 98.79 & 98.70 & 98.67 & 98.70 \\
\hline LZ206 & 99.12 & 98.76 & 98.72 & 98.70 & 100.00 & 99.27 & 98.73 & 98.69 & 98.51 & 98.66 & 99.71 \\
\hline LZ227 & 98.88 & 98.71 & 98.83 & 98.80 & 99.27 & 100.00 & 98.67 & 98.66 & 98.40 & 98.66 & 99.31 \\
\hline LZ95 & 98.80 & 99.07 & 98.96 & 98.74 & 98.73 & 98.66 & 100.00 & 98.93 & 98.71 & 99.89 & 98.83 \\
\hline WCFS1 & 98.76 & 99.00 & 98.95 & 98.79 & 98.69 & 98.66 & 98.93 & 100.00 & 98.71 & 98.99 & 98.77 \\
\hline J26 & 98.53 & 98.73 & 99.30 & 98.70 & 98.51 & 98.40 & 98.71 & 98.71 & 100.00 & 98.62 & 98.56 \\
\hline ST-III & 98.73 & 99.00 & 98.87 & 98.67 & 98.66 & 98.66 & 99.89 & 98.99 & 98.62 & 100.00 & 98.73 \\
\hline ZJ316 & 99.11 & 98.75 & 98.75 & 98.70 & 99.71 & 99.31 & 98.83 & 98.77 & 98.56 & 98.73 & 100.00 \\
\hline
\end{tabular}



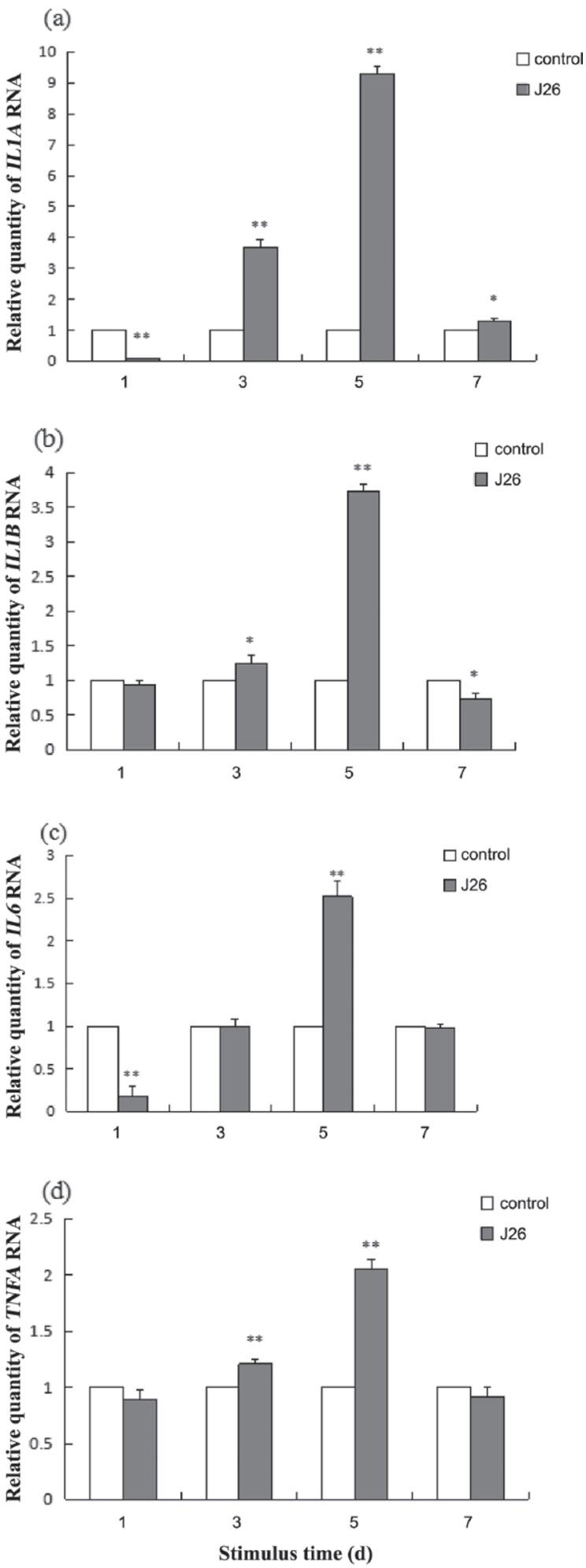

Figure 3. Expression of $I L 1 A, I L 1 B, I L 6$, and TNFA genes induced by Lactobacillus plantarum J26 in mice. Mice were intragastrically administered $0.3 \mathrm{~mL}$ of $L$. plantarum J26 (gray bars) or an equal amount of sterilized skim milk (white bars) for $7 \mathrm{~d}$, and the expression of $I L 1 \mathrm{~A}$ (a), IL1B (b), IL6 (c), and TNFA (d) was determined. Results are expressed as mean $\pm \mathrm{SD}$ of 3 independent determinations. ${ }^{*} P<0.05$, $* * P<0.01$ compared with control. and $\operatorname{tag} B 2)$ instead, and $\operatorname{tagB1}$ and tagB2 found in $L$. plantarum were supposed as tarK and tarL. The tarI and tarJ genes were annotated as D-ribitol-5-phosphate cytidylyltransferase and ribitol-5-phosphate 2-dehydrogenase. Hence, we propose that L. plantarum J26 contains tarI-tarL region and synthesizes ribitol-type backbones of WTA. Besides, 2 TA transporters (permease protein, tag; and ATP-binding protein, $\operatorname{tag} H$ ), subunits of the $\mathrm{ABC}$ transporter complex, were identified and may be associated with the immunomodulatory effects of L. plantarum J26.

In the current study, we demonstrated that the $L$. plantarum J26 is a probiotic strain with immunomodulatory activity (regulating the expression of immunerelated genes). The genome data of $L$. plantarum J26 provide substantial information for understanding the genetic basis of its probiotic properties. In addition, we discovered genes involved in key immunomodulatory molecules (teichoic acids) in the genome of J26. These results contribute to the development of immune-related functional dairy foods containing lactic acid bacteria. Further investigation into the molecular mechanism by which the TA-related gene products affect the host immune response are ongoing. The complete genomic sequence of $L$. plantarum J26 has been deposited in National Center for Biotechnology Information under GenBank accession nos. CP033616, CP033617, CP033618, CP033619, and CP033620.

\section{ACKNOWLEDGMENTS}

This work was supported by the National Natural Science Foundation of China (Beijing; project number 31871828) and the National High-Level Talents Special Support Program of China (810098).

\section{REFERENCES}

Barreto, F. M., A. N. Colado Simao, H. K. Morimoto, M. A. Batisti Lozovoy, I. Dichi, and L. Helena da Silva Miglioranza. 2014. Beneficial effects of Lactobacillus plantarum on glycemia and homocysteine levels in postmenopausal women with metabolic syndrome. Nutrition 30:939-942.

Chiu, Y.-H., Y.-C. Lu, C.-C. Ou, S.-L. Lin, C.-C. Tsai, C.-T. Huang, and M.-Y. Lin. 2013. Lactobacillus plantarum MYL26 induces endotoxin tolerance phenotype in Caco-2 cells. BMC Microbiol. $13: 190$.

Choi, S.-W., H.-N. Youn, W. Hong, J.-K. Park, S.-S. Yuk, J.-H. Kwon, J.-Y. Noh, J.-S. Kang, K.-J. Cho, J.-J. Ryu, J.-B. Lee, S.-Y. Park, I.-S. Choi, S.-W. Lee, and C.-S. Song. 2015. Intranasal administration model for evaluating protection against influenza virus in mice. J. Bacteriol. Virol. 45:44.

Coil, D., G. Jospin, and A. E. Darling. 2015. A5-miseq: An updated pipeline to assemble microbial genomes from Illumina MiSeq data. Bioinformatics 31:587-589.

Delcher, A. L., S. L. Salzberg, and A. M. Phillippy. 2003. Using MUMmer to identify similar regions in large sequence sets. Curr. Protoc. Bioinformatics 10.3.1-10.3.18. 10.1002/0471250953.bi1003s00. 
Gardner, P. P., J. Daub, J. G. Tate, E. P. Nawrocki, D. L. Kolbe, S. Lindgreen, A. C. Wilkinson, R. D. Finn, S. Griffiths-Jones, S. R. Eddy, and A. Bateman. 2009. Rfam: Updates to the RNA families database. Nucleic Acids Res. 37(Suppl. 1):D136-D140.

Goossens, D., D. Jonkers, M. Russel, E. Stobberingh, A. Van Den Bogaard, and R. Stockbrügger. 2003. The effect of Lactobacillus plantarum $299 \mathrm{v}$ on the bacterial composition and metabolic activity in faeces of healthy volunteers: A placebo-controlled study on the onset and duration of effects. Aliment. Pharmacol. Ther. 18:495-505.

Han, S. H., J. H. Kim, M. Martin, S. M. Michalek, and M. H. Nahm. 2003. Pneumococcal lipoteichoic acid (lta) is not as potent as staphylococcal lta in stimulating toll-like receptor 2 . Infect. Immun. 71:5541-5548.

Hou, Y., X. Li, X. Liu, Y. Zhang, W. Zhang, C. Man, and Y. Jiang. 2019. Transcriptomic responses of Caco-2 cells to Lactobacillus rhamnosus GG and Lactobacillus plantarum J26 against oxidative stress. J. Dairy Sci. 102:7684-7696. https://doi.org/10.3168/jds .2019-16332.

Hugenholtz, P. 1998. The Genera of Lactic Acid Bacteria. Blackie Academic and Professional, London, UK.

Jiang, Y., L. Li, H. Sun, Y. Shan, Y. Liu, L. Li, B. Qu, and C. Man 2016. Induction of cytokines via NF- $\mathrm{BB}$ and p38 MAP kinase signalling pathways associated with the immunomodulation by Lactobacillus plantarum NDC 75017 in vitro and in vivo. J. Funct. Foods 20:215-225.

Koren, S., B. P. Walenz, K. Berlin, J. R. Miller, N. H. Bergman, and A. M. Phillippy. 2017. Canu: Scalable and accurate long-read assembly via adaptive k-mer weighting and repeat separation. Genome Res. 27:722-736.

Kumar, S., G. Stecher, and K. Tamura. 2016. MEGA7: Molecular Evolutionary Genetics Analysis version 7.0 for bigger datasets. Mol. Biol. Evol. 33:1870-1874.

Lagesen, K., P. Hallin, E. A. Rodland, H. H. Staerfeldt, T. Rognes, and D. W. Ussery. 2007. RNAmmer: Consistent and rapid annotation of ribosomal RNA genes. Nucleic Acids Res. 35:3100-3108.

Lee, I., Y. Ouk Kim, S. C. Park, and J. Chun. 2016. OrthoANI: An improved algorithm and software for calculating average nucleotide identity. Int. J. Syst. Evol. Microbiol. 66:1100-1103.

Li, L., Y. J. Jiang, X. Y. Yang, Y. Liu, J. Y. Wang, and C. X. Man. 2014. Immunoregulatory effects on Caco-2 cells and mice of exopolysaccharides isolated from Lactobacillus acidophilus NCFM. Food Funct. 5:3261-3268.
Liu, W. H., C. H. Yang, C. T. Lin, S. W. Li, W. S. Cheng, Y. P. Jiang, C. C. Wu, C. H. Chang, and Y. C. Tsai. 2015. Genome architecture of Lactobacillus plantarum PS128, a probiotic strain with potential immunomodulatory activity. Gut Pathog. 7:22.

Lowe, T. M., and S. R. Eddy. 1997. tRNAscan-SE: A program for improved detection of transfer RNA genes in genomic sequence. Nucleic Acids Res. 25:955-964.

Pruitt, K. D., T. Tatusova, G. R. Brown, and D. R. Maglott. 2012 NCBI Reference Sequences (RefSeq): Current status, new features and genome annotation policy. Nucleic Acids Res. 40:D130-D135.

Round, J. L., and S. K. Mazmanian. 2009. The gut microbiota shapes intestinal immune responses during health and disease. Nat. Rev. Immunol. 9:313-323.

Salvetti, E., S. Torriani, and G. E. Felis. 2012. The genus Lactobacillus: A taxonomic update. Probiotics Antimicrob. Proteins 4:217-226.

Smelt, M. J., B. J. de Haan, P. A. Bron, I. van Swam, M. Meijerink, J. M. Wells, M. Kleerebezem, M. M. Faas, and P. de Vos. 2013 The impact of Lactobacillus plantarum WCFS1 teichoic acid Dalanylation on the generation of effector and regulatory T-cells in healthy mice. PLoS One 8:e63099.

Sun, Z., H. M. Harris, A. McCann, C. Guo, S. Argimon, W. Zhang, X. Yang, I. B. Jeffery, J. C. Cooney, T. F. Kagawa, W. Liu, Y. Song, E. Salvetti, A. Wrobel, P. Rasinkangas, J. Parkhill, M. C. Rea, O. O'Sullivan, J. Ritari, F. P. Douillard, R. Paul Ross, R. Yang, A. E. Briner, G. E. Felis, W. M. de Vos, R. Barrangou, T. R. Klaenhammer, P. W. Caufield, Y. Cui, H. Zhang, and P. W. O'Toole. 2015. Expanding the biotechnology potential of lactobacilli through comparative genomics of 213 strains and associated genera. Nat. Commun. 6:8322.

Walker, B. J., T. Abeel, T. Shea, M. Priest, A. Abouelliel, S. Sakthikumar, C. A. Cuomo, Q. Zeng, J. Wortman, S. K. Young, and A. M. Earl. 2014. Pilon: An integrated tool for comprehensive microbial variant detection and genome assembly improvement. PLoS One 9:e112963.

\section{ORCIDS}

Ziwei Zhang @ https://orcid.org/0000-0002-7070-0067

Chaoxin Man (®) https://orcid.org/0000-0003-2050-4639

Yujun Jiang (ㄴ) https://orcid.org/0000-0002-2857-2137 